\title{
Quetiapine induced acute coronary syndrome: Possible Kounis syndrome.
}

\author{
Çağlar Kaya*, Servet Altay \\ Department of Cardiology, Trakya University, Edirne, Turkey.
}

\begin{abstract}
There are a lot of reasons for the chest pain and acute coronary syndrome. The acute coronary syndrome occurs by a coronary vasospasm with an allergic reaction which is defined as Kounis Syndrome (KS). A 28 year old male patient was consulted to the emergency department with dyspnea resembling asthma, rare urticarial rashes, and violent chest pain. In the electrocardiogram, there was ST segment elevation at the D2-D3-AvF derivations. An urgent coronary angiography was performed resulting in the diagnosis of inferior myocardial infarction. A Normal coronary anatomy was detected. By the detailed history, we got that the patient was a smoker and the patient had taken $200 \mathrm{mg}$ of Quetiapine to sleep. Only dyspnea and minimal rashes were detected for the allergic symptoms. With these signs and symptoms, the diagnosis was thought to be Quetiapine-induced Kounis Syndrome. In Kounis syndrome, the major pathophysiological mechanism of the coronary spasm is an allergic reaction took place due to the inflammatory mediators which discharged during a hypersensitivity reaction triggered by food, insect bites or drugs. Here, we report a case of coronary spasm due to the allergic reaction followed by the intake of Quetiapine.
\end{abstract}

Keywords: Allergic angina, Coronary vasospasm, Hypersensitivity, Kounis syndrome.

Accepted on October $24^{\text {th }}, 2018$

\section{Introduction}

Kounis syndrome is a disease in which sudden coronary vasospasm and acute coronary syndrome are present at the same time of hypersensitivity [1]. This disease is more common in male and middle age groups [1]. Although KS is a wellestablished disease in the cardiology practice, many patients still cannot be diagnosed because of various reasons such as insufficient clinician awareness, less questioning of allergen exposure. Kounis syndrome should be taken into account in young, healthy patients with no cardiac risk factors which are observed with acute coronary syndrome after administration of a potentially allergic agent. As known Quetiapine is used in the treatment of various diseases such as bipolar disorder, schizophrenia and major depressive disorder [2]. In this article, we have discussed a 28 year old male patient who had Kounis syndrome set off oral Quetiapine intake (to sleep) without having the typical allergic symptoms.

\section{Case Report}

In the spring period, in March, a 28 year old male patient was applied to the emergency department of Edirne Trakya University Hospital with dyspnea resembling asthma, rare urticarial rashes, and typical severe chest pain. The patient stated that these complaints were the first time in his life. The complications continued for $40 \mathrm{~min}$ and the pain was extending to his back. At the first evaluation, the pain was continuous, his blood pressure was 110/65, and his heart rate was $77 \mathrm{bpm}$. Physical examination showeda man with a body weight of 73 $\mathrm{kg}$, height $174 \mathrm{~cm}$ with (Body Mass Index) BMI $24.11 \mathrm{~kg} /$ $\mathrm{m}^{2}$. The pulmonary examination was normal and there was no abnormality in the abdominal examination. In the ECG, there was an ST segment elevation at the D2-D3-AvF (Figure 1). The patient was diagnosed with inferior MI, the patient was treated with the $300 \mathrm{mg}$ of acetylsalicylic acid, $600 \mathrm{mg}$ of clopidogrel, 5000 IU of unfractionated heparin, and an immediate coronary angiography was performed. Normal coronary arteries were determined (Figure 2). Because of the acute coronary syndrome, we didn't medicate the patient with the agents that provokes vasospasm. After the treatment of the chest pain, a new ECG was taken, and we saw that the ST segment elevations had disappeared a bit compared to the first ECG (Figure 3).

Echocardiography of the patient was normal. Cardiac markers such as troponin I and creatinine kinase-MB revealed no pathologic findings. From the detailed anamnesis, we learned that the patient was a smoker ( $>10$ cigarettes per a day), no alcohol using and had a history of allergic asthma in childhood. His family didn't have a history of any cardiac disease or allergy. The patient has taken $200 \mathrm{mg}$ of Quetiapine to sleep. With the history of allergic asthma, rashes, normal angiography, and triggering drug intake were thought to be the reasons for KS induced by Quetiapine. We tracked the patients' situation; cardiac biomarkers did not increase. Antihistaminic drugs and steroids were not given because of the spontaneous remission of dyspnea and urticarial rash. The patient was suggested to quit smoking and use $100 \mathrm{mg}$ of acetylsalicylic acid.

\section{Discussion}

Kounis and Zavras described the concept of allergic angina in 1991 [3]. Kounis Syndrome is a disease that occurs after an allergic event that causes vasospastic angina or acute coronary syndrome. Some inflammatory and vasoactive mediators which released during the allergic reaction are responsible 


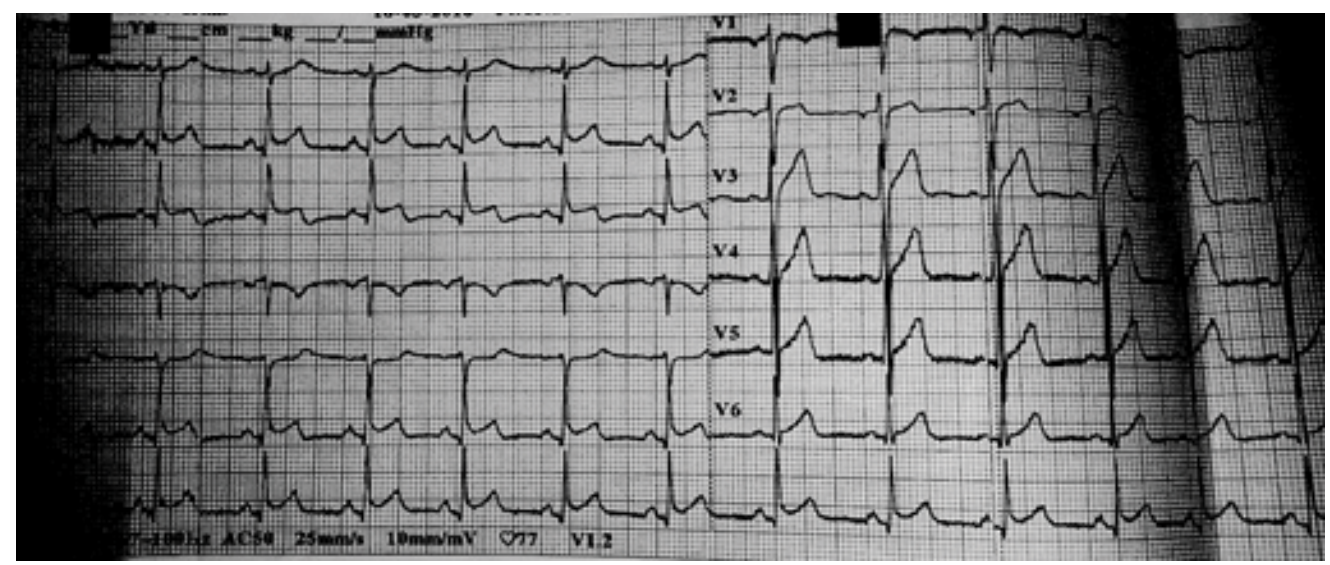

Figure 1: ECG shows the ST segment elevation at the D2-D3-AvF.

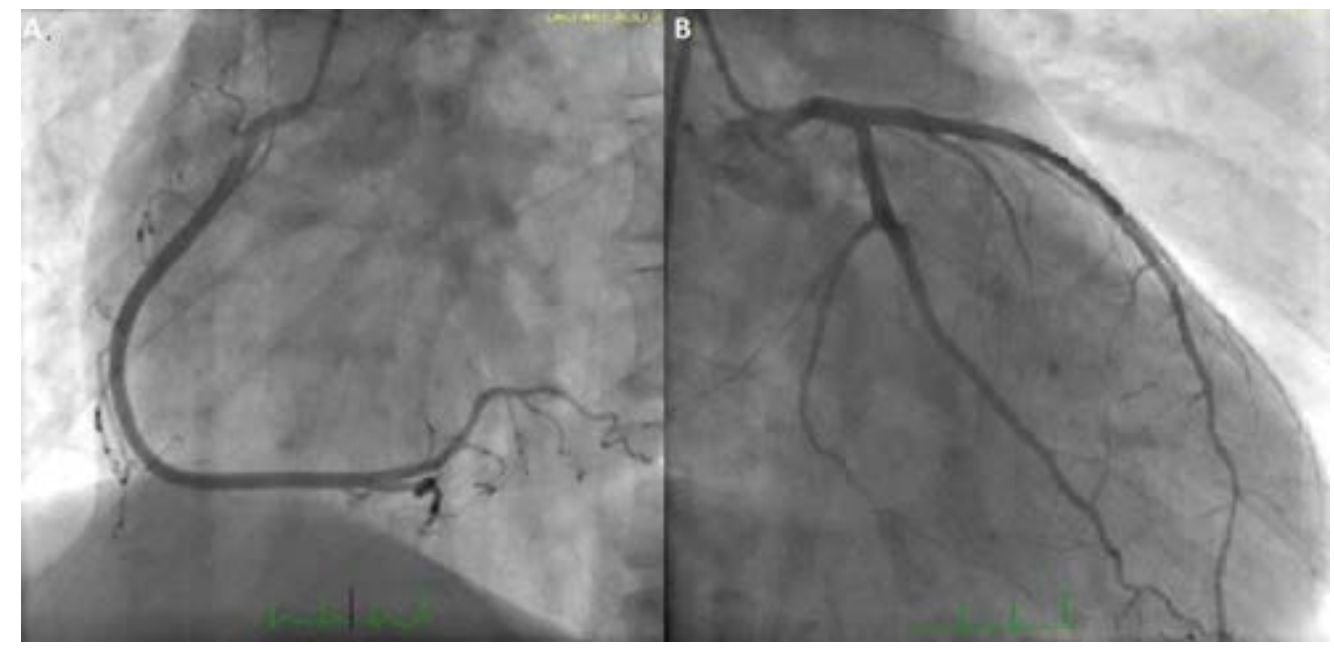

Figure 2: The coronary angiography, which was performed at the time of chest pain, revealed normal coronary arteries.

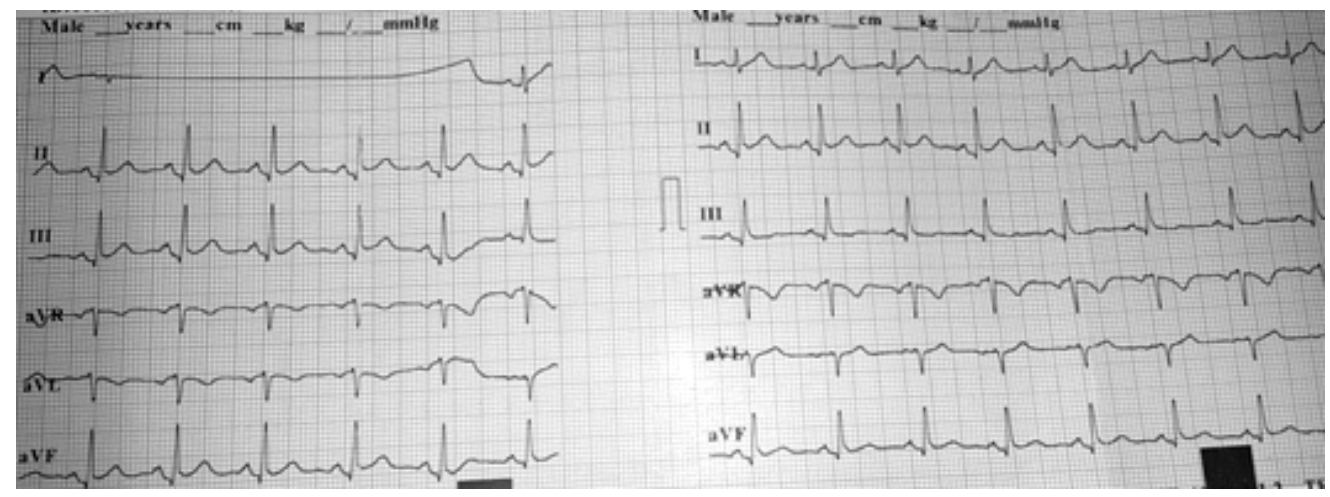

Figure 3: Resolution of ST segment elevations.

for the pathophysiology of this syndrome [4]. The coronary artery vasospasm which occurs after the release of vasoactive mediators is the mechanism of this syndrome [5]. Some of these mediators are histamine, serotonin, platelet activating factor, chemokines, leucotrienes, and prostaglandins. Histamine, serotonin and leukotrienes are the most important mediators which could be responsible for coronary vasospasm and Kounis Syndrome [5,6]. Endothelial dysfunction is triggered by these substances causing coronary vasospasm [5,7]. Several factors or agents that cause kounis syndrome have been reported in the literature. These etiologies include a plenty of drugs, various conditions and environmental exposures [7,8]. For instance: insect bites, asthma, latex, antibiotics, some foods, painkillers, bee stings [7]. In some studies and databases, NSAIDs are the most common drugs that cause Kounis Syndrome among drugs [9]. Kounis syndrome could be observed in three variants. Type 1 observed in patients with no atherosclerosis, type 2 observed in patients who have previous atherosclerosis, and type 3 occurs after drug-eluting stent implantation in recent years [10]. Kounis Syndrome, which is a common disease, may show similarities in different diagnoses such as Prinzmetal Angina (PA). The critical point is to make diagnose to this disease. Diagnosis in Kounis syndrome is based on history, imaging tests and clinical manifestations [11]. Tryptase level, selective spasm test with acetylcholine or ergonovine and skin-prick allergic test can be used for diagnosis [11]. In this patient, although ischemic ST 
interval and $\mathrm{T}$ wave changes have been observed, no increase in cardiac biomarker is seen. The clinical presentation did not progress through the myocardial infarction. The patient had taken a drug for the first time and he used a high dose as a beginning treatment. This leads us to consider a probable etiological factor for the reason of this disease. Therefore, a detailed history was taken from the patient and as a result, Quetiapine intake was detected. The diagnosis of KS wasn't certain because the patient had no anaphylactic symptoms except asthma-like dyspnea and minimal rashes. On the other hand, at the initial evaluation, there was a young patient with the acute coronary syndrome who has chest pain and asthma-like dyspnea after a suspected drug usage, and this clinical case leads us to consider the diagnosis of the Kounis Syndrome primarily. In this article, we pointed out the fact that Quetiapine triggers acute coronary syndrome in Quetiapine sensitive people. There are many cases of drug induced KS that have been reported. According to our investigations, the relationship of Kounis Syndrome and Acute Coronary syndrome with Quetiapine was not clearly stated in the literature. We thought of Kounis Syndrome because of the fact that our patient was young, had normal coronary artery, didn't have a serious cardiovascular risk except smoking and developed this clinical picture after drug intake. In conclusion, a detailed history should be reported for every patient who has the acute coronary syndrome. According to such detailed history, clinicians determine the treatment and follow-up the procedures. After discharge, the patients should go to the immunology and cardiology outpatient clinics. It should be noted that unnecessary revascularization can be prevented by reporting the detailed history, right diagnosis, and correct treatment.

\section{REFERENCES}

1. Abdelghany M, Subedi R, Shah S, et al. Kounis syndrome: A review article on epidemiology, diagnostic findings, management and complications of allergic acute coronary syndrome. Int J Cardiol. 2017:232;1-4.
2. Sadock BJ. Kaplan and sadock's synopsis of psychiatry: Behavioral sciences/clinical psychiatry. Indian J Psychiatry. 2009:51(4);331.

3. Kounis NG, Zavras GM. Histamine-induced coronary arteryspasm: The concept of allergicangina. Br J ClinPract. 1991:45(2);121-8.

4. Kounis NG, Hahalis G, Manola A, et al. Kounis syndrome (allergic angina and allergic myocardial infarction). Nova Science Publishers. 2008

5. Altay S, Cakmak HA, Erer B, et al. The allergic angina syndrome in naproxen sodium induced type 1 hypersensitivity reaction in an allergic asthmatic young woman: Kounis syndrome. Acta card sin. 2012: 28(2);152-6.

6. Genovese A and Spadaro G. Highlights in cardiovascular effects of histamine and H1-receptor antagonists. Allergy. 1997:52(34);67-78.

7. Kounis NG. Kounissyndrome (allergicangina and allergicmyo- cardialinfarction): A natural paradigm? Int $\mathrm{J}$ Cardiol. 2006:7(1);7-14.

8. Yang HP, Chen FC, Chen CC, et al. Manifestations mimicking acute myocardial infarction after honey bee sting. Acta Cardiol Sin. 2009:25;31-5.

9. Renda F, Landoni G, Trotta F, et al. Kounis syndrome: An analysis of spontaneous reports from international pharmacovigilance database. Int J Cardiol. 2016:203;217-20.

10. Kounis NG, Mazarakis A, Tsigkas G, et al. Kounis syndrome: A new twist on an old disease. Future Cardiol. 2011:7(6);805-24.

11. Pradhan S, Christ M, Trappe H-J. Kounis syndrome induced by amoxicillin following vasospastic coronary event in a 22-year-old patient: a case report. Cardiovasc Diagn Ther. 2018:8(2);180-185.

\section{*Correspondence to:}

Çağlar Kaya

Department of Cardiology, Trakya University, Edirne, Turkey

E-mail: caglarkaya2626@gmail.com 